\title{
Multichannel Selective Linear Prediction Spectrum Analysis for Detection of Cardiac Late potentials
}

\author{
U.C. Niranjan and I.S.N. Murthy
}

Department of Elec. Engg., Indian Institute of Science, Bangalore 560 012, INDIA.

\begin{abstract}
In this peper we propose a new technique for the frequency analysis of the signal averaged electrocardiogram (SABCG), based on a paranetric codel. A part of the spectrue of interest is characterised by a selective linear prediction (SLP)eode1. A wultichannel extension to the SLP wethod is proposed to analyze all the three orthogonal lead data at one stroke. Consideration of three channel sarcc as a vector process results in matrix predictor coefficients and spectral matrix containing auto and cross spectra of individual channels. Vector sum of the area under the model auto spectra is used in the detection of Late Ventricular potentials. In addition, the coherence function represents the asount of correlation between channels over the frequency of interest.
\end{abstract}

\section{INTRODUCTION}

The cardiac late potentials are high frequency $(60-180 \mathrm{~Hz})$, low amplitude (1$20 \mu v)$, fragmented and delayed signal components found at the end of the QRS complex in myocardium infarcted patients prone to life threatening ventricular tachycardia [1]. Classification of these potentials from the surface ECG constitutes an important signal detection problem. Traditional approaches such as matched filtering and short time Fourier transform and more recently, improved time-frequency representation of signals such as wavelet transform and wigner ville distribution are used to characterize late ventricular potentials (LVP) [2]. Spectral leakage, choice of time shift and frequency dilation parameters and cross terms are some of the limitations of these techniques.

In this paper we attempt to improve the frequency resolution of LVP components in the spectrum using a selective linear prediction (SLP) model. A multichannel extension to the SLP technique is proposed, to incorporate the time correlations between individual signal averaged electrocardiograms (SAECG) into the model. The presence of Late potentials is inferred from an area parameter derived from the model spectra matrix.

\section{METHOD}

1) Multichannel Linear prediction analysis: The multichannel linear prediction (LP) model represents the signal vector $x(n)$ as a linear combination of its past $p$ samples and a residual error vector $\underline{\epsilon}(\mathrm{n})$, i.e.

$$
\underline{\underline{x}}(\mathbf{n})=\sum_{i=1}^{p} A(i) \underline{x}(n-i)+\underline{E}(n)
$$

where $A(i)$ is the ith predictor coefficient matrix of size $\mathrm{m} \times \mathrm{m}, \mathrm{m}$ being the number of channels [3].

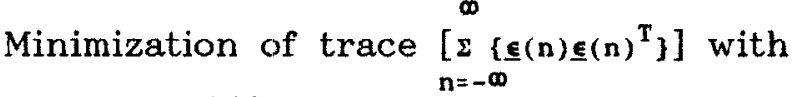

respect to $A(i)$ 's results in the Yule-Walker equation,

$$
\sum_{i=1}^{p} R_{\underline{X x}}(k-i) A^{T}(i)=R_{\underline{x x}}(k), k=1,2, \ldots p
$$

where $R_{1}(\mathrm{k})$ is the autocorrelation matrix of the vector process $x(n)$ at lag $k$.

2) Selective linear prediction: Here, the portion of the signal spectrum to be modelled is first translated to the angular frequency 0 to $\pi$ radians [4]. Autocorrelatión coefficients are then computed from the evenly mapped spectrum and used in (2) to estimate the model parameters. Estimate of the spectral matrix, based on the multichannel SLP model is then given by

$$
P(f)=A^{*-1}(f) \sigma_{E} A(f)
$$

where $A(f)$ is the Fourier transform of the predictor coefficient sequence and $\sigma$ is the prediction error power matrix. The difagonal elements of $P(f)$ are the power spectral densities (PSDs) of the individual channels, while the off-diagonal elements represent the cross-PSD between them.

\section{Results}

We now present the results of analysis of three lead SAECG obtained from a patient with documented ventricular tachycardia (VT). The averaged signals with a sampling rate of $1000 \mathrm{~Hz}$ and duration $600 \mathrm{~ms}$ are shown in Figs. a-c. The auto spectra of the hamming window weighted signals, obtained from the fast Fourier transform (FFT) are shown in Figs. $d-f$. All the spectra are plotted over the frequency range 60-180 $\mathrm{Hz}$, in log magnitude. The multichannel LP model was then estimated using the autocorrelation functions obtained from these auto-spectra as well cross-spectra computed from FFT over the same frequency range. Akaike's information criterion (AIC) estimated a model order of 10 for this particular multichannel signal [3]. The diagonal elements of the model spectral matrix are shown in Figs. g-i, which displays sharper peaks and brings out the 
finer details, dormant in the FFT spectra. Also, the valleys in the signal spectra are reproduced accurately, since the transfer function of a multichannel LP model is rational, containing zeros along with poles. The magnitude squared coherence, computed from the self and cross spectra of channels 1,$2 ; 2,3$ and 3,1 are shown in Figs. j-l. These plots represent the amount of correlation between individual channels, over the frequency of interest. Vector sum of the areas under the model auto spectra computed by trapezoidal rule, was found to be 10192. This parameter is well above 8431 , obtained in case of subjects without $\mathrm{VT}$ and thus promises to be a reliable tool for detection of LVP.

\section{Discussion \& Conclusron}

Selective linear prediction technique represents only the desired part of the frequency spectrum, thus increasing the spectral dynamic range and resolution with a model of reduced order. The model spectra can be computed inside unit circle to further enhance the dynamic range. Also the location of the spectral peaks can be obtained from the resonant frequencies of the model poles. Analysis of the three

channel signal of length 600 samples requires 2.5 MFLOPs. By considering different segments of signal near QRS complex, the commonly used spectral area ratios can be used for more robust detection of LVPs.

A simple, new technique is presented for the detection of LVPs in multichannel SAECG signal, based on a selective linear prediction model. The results of analysis are highly satisfactory.

\section{REFERENCES}

[1] H. F. Schels, R. Haberl, G. Jilge, P. Steinbigler and G. Steinbeck, "Frequency analysis of the electrocardiogram with maximum entropy method for identification of patients with sustained ventri cular tachycardia", IEEE Trans. BME, vol. 38, pp. 821-826, September 1991 .

[2] M. S. Fuller, T. Dustman and R. Freedman, "Wave let analysis of the signal averaged electrocar diogram", Proc. of 13 th annual IEEE - EMBS confe rence, Orlando, USA, pp. 639-640, 1991.

[3] S. M. Kay, Modern spectral estimation, New Jer sey: Prentice Hall, 1988, pp. 446-478.

[4] D. Nandagopal, J. Mazumdar and R.E. Bogner, "Spectral analysis of second heart sound in normal children by selective linear prediction coding", Med * Biol. Engg \& Comput., pp 229-239, May 1984.
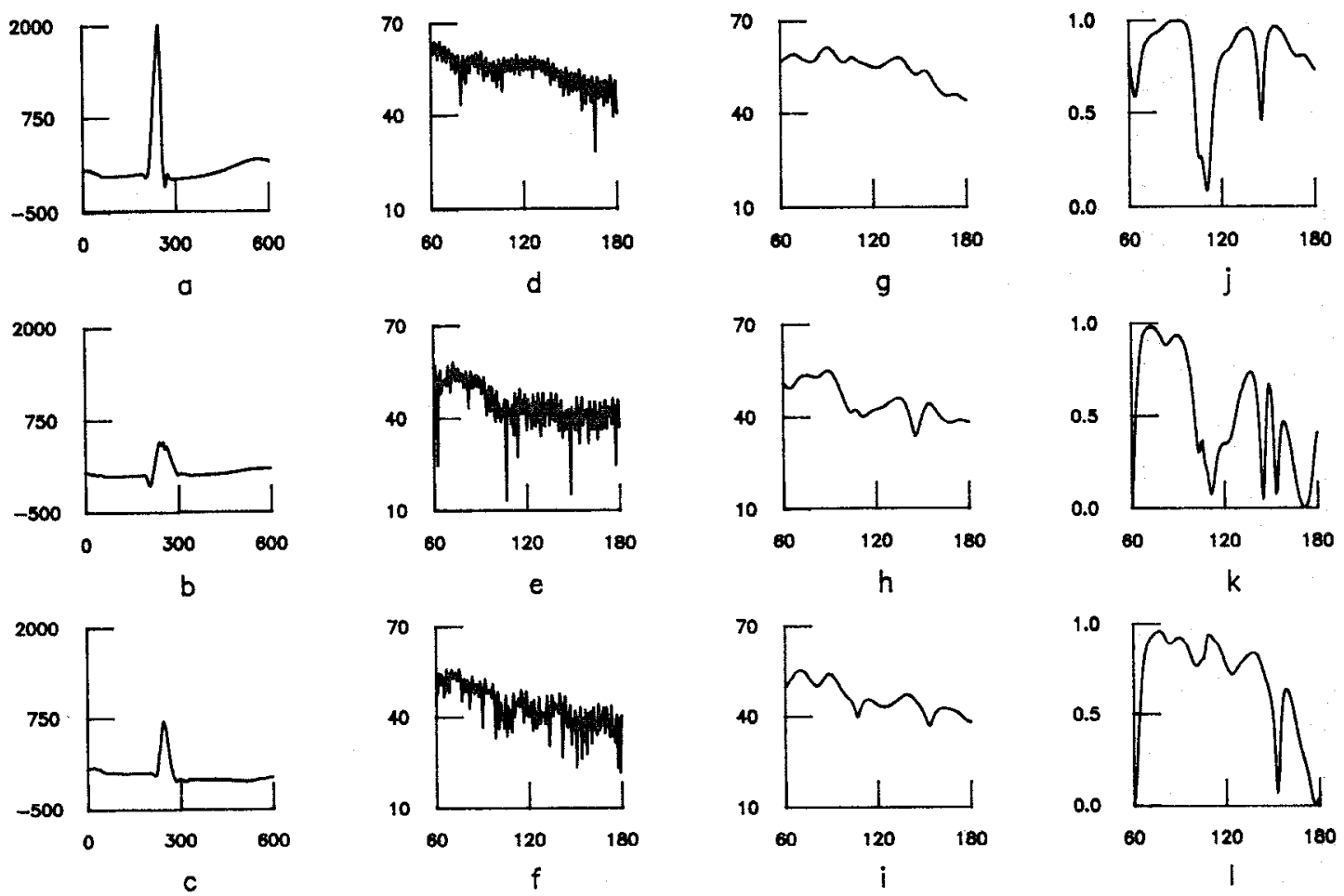

Figs. $a-c$ : 3 lead SAECG, $d-f$ and $g-i$ : auto spectro from FFT and SLP methods, j-l: coherence functions.

$X$ axis: Figs. a-c: sample no, $d-l$ : frequency in $\mathrm{Hz}$.

$Y$ axis: Figs. a-c: amplitude, $d-i$ : decibels, $j-l$ : magnitude. 